\title{
Current status on single molecular sequencing based on protein nanopores
}

\author{
Jiawei Shen, Yongyong Shi \\ College of Biomedical Engineering, Shanghai JiaoTong University, 800Dongchuan Road, Shanghai200243, P. R. China \\ * Corresponding author: shiyongyong@gmail.com(Yongyong Shi) Tel/fax.: 86-021-62933338
}

\begin{abstract}
In the 1960s, Hladky and Haydon have succeeded in detecting ionic flows across thin lipid membrane when adding small lipid-soluble molecules such as surface-active polypeptides to artificial membrane, which has laid the foundation for decoding single strand DNA when it crosses the nanopore under an applied electric field. In this paper, we will review several kinds of protein nanopores used in single molecular sequencing, such as $\alpha$-hemolysin nanopore, MspA nanopore and membrane-adapted phi29 motor protein nanopore.
\end{abstract}

Keywords: Single Molecular Sequencing, Nanopore, $\alpha$-hemolysin, MspA, Phi29 motor

Citation: J Shen, et al. Current status on single molecular sequencing based on protein nanopores. Nano Biomed. Eng. 2012, 4(1), 1-5 DOI: $10.5101 /$ nbe.v4i1.p1-5

\section{Introduction}

A simple idea for single molecular sequencing is that let nanometer-scale pore $(\sim 1.3 \mathrm{~nm}$ in diameter $)$ provide the sole pathway for single-stranded DNA or RNA. When single-stranded DNA or RNA is driven through the pore under an applied electric field, the ionic current through the pore is reduced and every single nucleotide generates a characteristic residual ionic current which corresponds to its DNA sequence. Theoretically, this method can be used to detect the DNA or RNA sequences rapidly. Based on this idea, several nanopores have been reported to succeed in detecting DNA/RNA sequences. In this paper, we will review some of these nanopores, thus providing readers with a brief framework of the nanopore sequencing.

\section{1. $\alpha$-hemolysinnanopore}

The first used nanopore in single molecular sequencing is the $\alpha$-hemolysinnanopore. It is a $33 \mathrm{kD}$ protein that is isolated from Staphylococcus aureus, which undergoes self-assembling in lipid bilayers and finally forms a channel with a relatively large nanopore [1].It forms a homo-heptameric beta-barrel within biological membranes. This may span the membranebarrier, and a broader segment (called the "mushroom cap") which extends into one of the aqueous compartments [2]. Its total length is about $100 \AA$. The structure of $\alpha$-Hemolysin is showed in Fig. 1.

Mark Akeson et al.have demonstrated that nanopore formed by $\alpha$-Hemolysincan act as a detector that can discriminate between pyrimidine and purine segments rapidly along RNA molecule [4]. The apparatus are shown in Fig. 2. They use a U-shaped Teflon path tube connecting two $70-\mu l$ baths milled into a Teflon support while the baths and the Telflontuble are filled with $1 \mathrm{M} \mathrm{KCL}$ buffer. These baths are then connected to an Axopatch 200B amplifier by the Ag-AgCl electrodes. One end of the Teflon path tube has a conical tip which narrows abruptly to a $25-\mu \mathrm{m}$ conical aperture. The diphytanoyl phosphatidylcholine/hexadecane bilayers are formed across this aperture. one or more $\alpha$-hemolysims are inserted into the bilayer. Nucleic acids are driven through the $\alpha$-hemolysin channel under an applied voltage of about $+120 \mathrm{mV}$ at the trans side[4].

The results are shown in Fig. 3. As predicted by the crystallographic model, the translocation of the ssDNA has caused an $85 \%$ blockade which corresponds to sequence of the ssDNA. These blockades could be amplified by PCR from the trans compartment. The polynucleotide blockade durations mainly depend on strand-length of the DNA, which shows that DNA traverses the pores with strand extended. That is, the requirement of single strand transport across a nanopore has been fulfilled. However, it still remains unknown that whether the DNA translocation through $\alpha$-hemolysin is unidirectional, and whether thermal fluctuations could cause the strands to diffuse backward randomly against 
http://nanobe.org

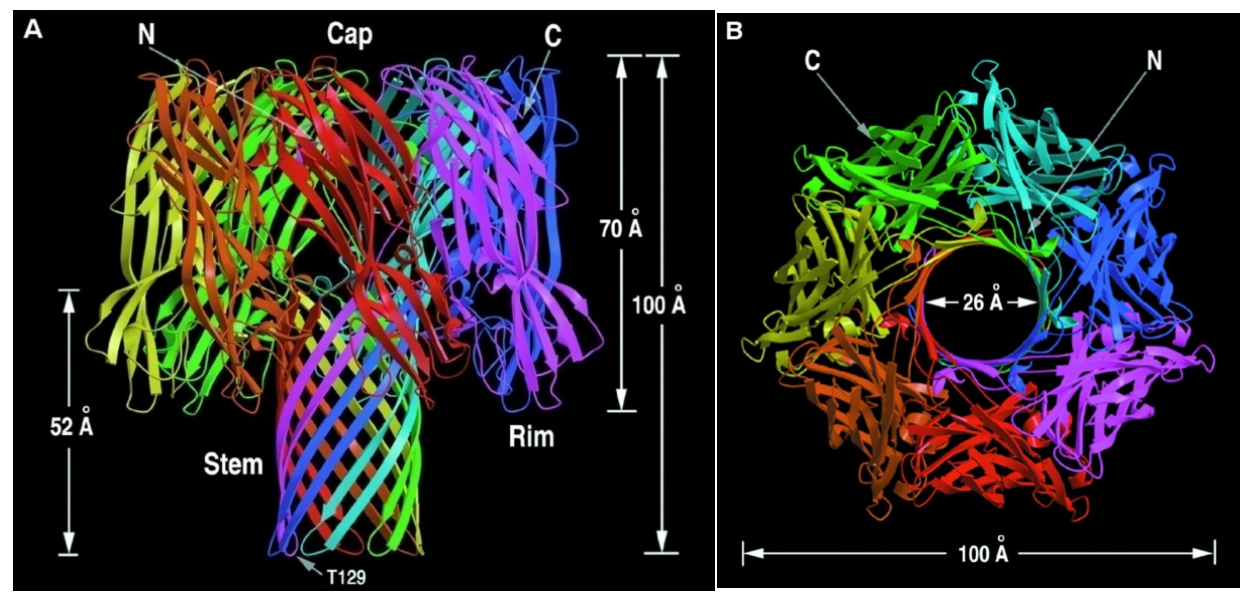

Fig. 1 Ribbon representations of the $\alpha$-HL heptamer with each protomer in a different color[3]

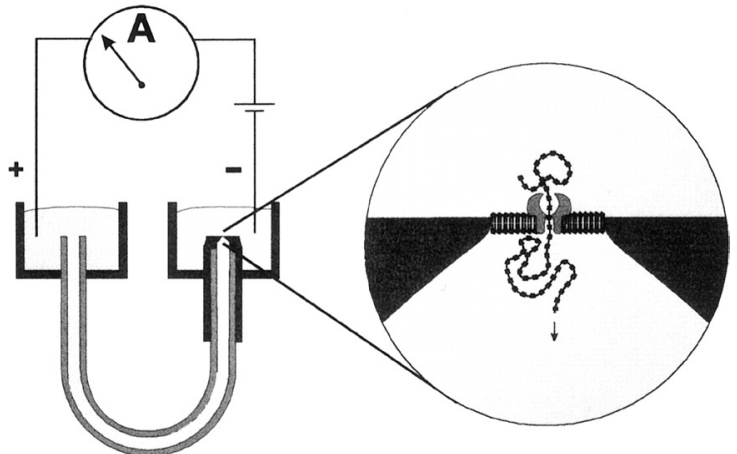

Fig. 2 Horizontal bilayer apparatus[4].

the electric field[2].

However, the speed of ssDNA transporting the nanopore is so high that this method can only identify between pyrimidine and purine rather than a single base of the ssDNA. Moreover, the nanopore can accommodate $\sim 15$ bases at the same time, which may cause signal confusion. Last but not least, the secondary structure of the single-stranded DNA and the nanopore is easy to aging and the experiment environment plays an important role, which can easily interfere the experimental condition.

\section{MspA nanopore}

$\alpha$-hemolysin's $5 \mathrm{~nm}$-long cylindrical beta barrelmay dilute the ion current which is specific to the single nucleotide and will generate a small current difference between nucleotides. This structural drawback is believed to be overcome by the channel protein Mycobacterium smegmatis porinA(MspA)[5]. MspA is a channel-forming protein which constitutes the major diffusion pathway for hydrophilic solutes in M. smegmatis. MspAhas remarkable stability when under strong environmental stresses. it can retain channel-forming activity even after exposure to any $\mathrm{pH}$ ranging from 0 to 14 , extraction at $100^{\circ} \mathrm{C}$ for about 30 minutes and further incubation at $80^{\circ} \mathrm{C}$ in the presence of $2 \%$ SDS for about 15 minutes. It can also be rationally modified based on its crystal structure. Furthermore, MspA has a narrow and short channel constriction that is very suitable for DNA sequencing [6]. The constriction is about 1-nm in total length and 1-nm in width. The geometry of MspA may provide improved spatial resolution. The characteristics of the ionic current blockades are mainly determined by the polynucleotide segment passing through the narrow region of the nanopore.

The wide-type MspA is initially negatively-charged, thus, preventing ssDNA translocation. Butler, T. Z., Pavlenok, M. et al[6] have used the site-directed mutagenesis to remove the three negatively-charged aspartic acids and replaced them with neutral asparagines. And the resulting mutant of MspA is called M1-NNNMspA which allows ssDNA translocation.

In order to overcome the problem that DNA translocation is so rapid, with the speed of $>10 \mathrm{nt} / \mu \mathrm{s}$ in solid state nanopores and $>1 \mathrm{nt} / \mu \mathrm{s}$ in $\alpha$-hemolysin and MspAnanopores,Derrington, I. M., Butler, T. Z. et al. [5] have proposed a method. That is, adding a section of double stranded DNA (called hairpin duplex) at the head of single stranded DNA(called hairpin tail which we are interested in can halt the translocation of the DNA and hold the single-stranded section of interests in the nanopore's constriction. At a driving voltage of about $180 \mathrm{mV}$, the hairpin duplex dissociates after $\sim 10 \mathrm{~ms}$. While the hairpin tail is held in the constriction of MspA, the measured ionic current primarily depends on the composition of the single-stranded DNA residing in the confining constriction. After the dissociation of the hairpin duplex, the DNA completes translocation into the lower potential chamber at the speed of faster than $1 \mathrm{nt} / \mu \mathrm{s}$. Thus, the ssDNA section can be sequenced in this way. 
Article
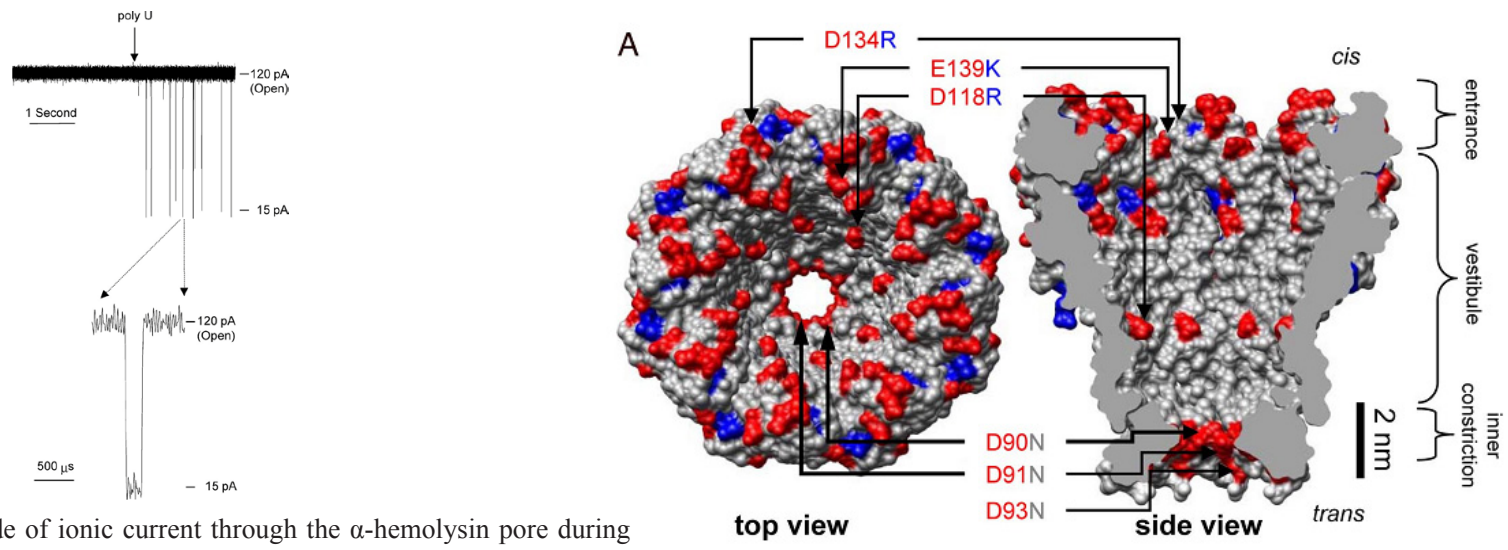

Fig. 3 Blockade of ionic current through the $\alpha$-hemolysin pore during the translocation of poly(U) RNA homopolymers. The poly $(\mathrm{U})$ strands is $150 \mathrm{nt}$ in length on average. The applied potential was about $120 \mathrm{mV}$ at room temperature. The buffer was $10 \mathrm{mM} \mathrm{HEPES} / \mathrm{KOH}$ and $1 \mathrm{M}$ KClat $\mathrm{pH} 8.0$ [2]
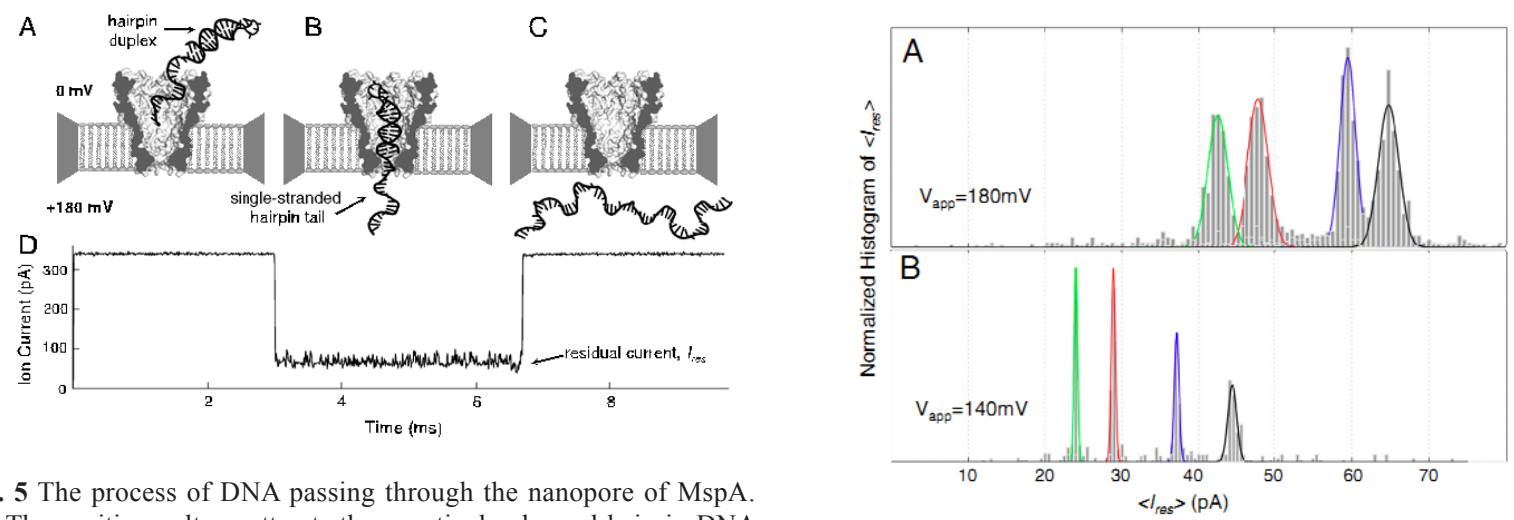

Fig. 5 The process of DNA passing through the nanopore of MspA. (A) The positive voltage attracts the negatively-charged hairpin DNA into the nanopore. (B) The DNA threads through the nanopore until the wilder hairpin duplex section of the DNA prevents further translocation. (C) After a couple of milliseconds, the hairpin duplex dissociation takes place and allows for complete translocation (D) the resulting current shows that the hairpin duplex of the DNA present in the nanopore allows a residual current until the hairpin duplex dissociates [5].

Fig. 4 The structure of MspA[6]

Fig. 6 Example histograms of the measured average residual ion current. The current is shown for different hopolymer single-stranded tails of a 14 base-pair hairpin. (A) under the voltage of $+180 \mathrm{mV}$ (B) under the voltage of $+140 \mathrm{mV}$. The reduction in widths at $+140 \mathrm{mV}$ is due to increased time averaging because the dissociation times are nearly 30 times longer than the dissociation times at $180 \mathrm{mV}[5]$.
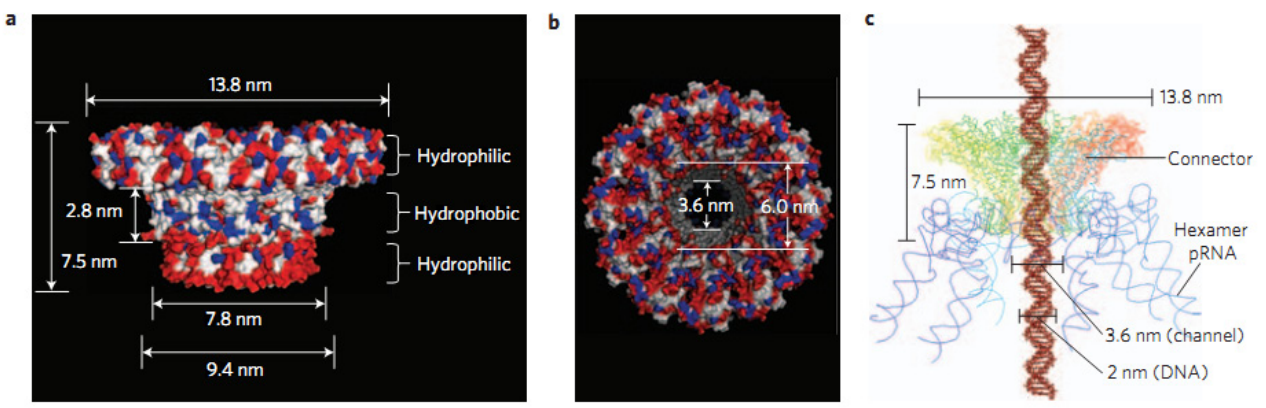

Fig. 7 The structure of the phi29. (a) Side view of the phi29 connector. The acidic is shown in red while the basic is shown in blue, and amino acids are shown in white. (b) Top view of the phi29 connector. (c)illustration of the entire phi29 DNA packaging motor showing DNA translocation through the connector[7].

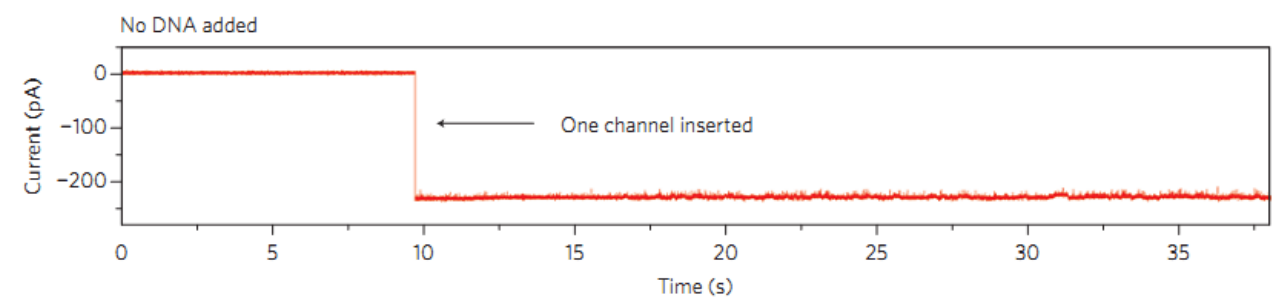

Fig. 8 (a) Translocation of double-stranded DNA through connector channels. (a) The current trace when no DNA is presented (control). 


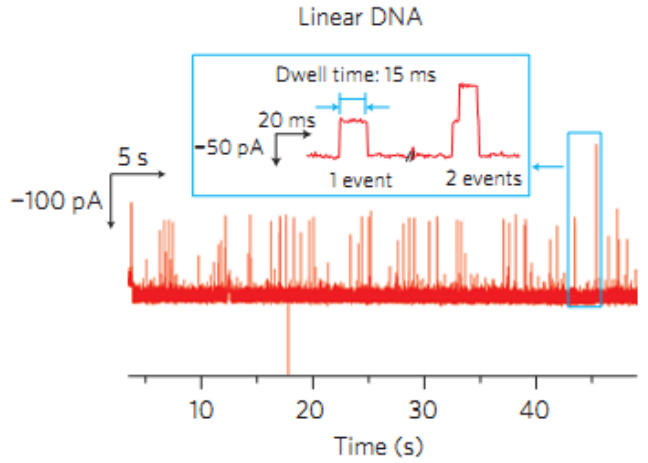

Fig. 8 (b) Blockades cased by 45 pM double-stranded linear plasmid DNA.
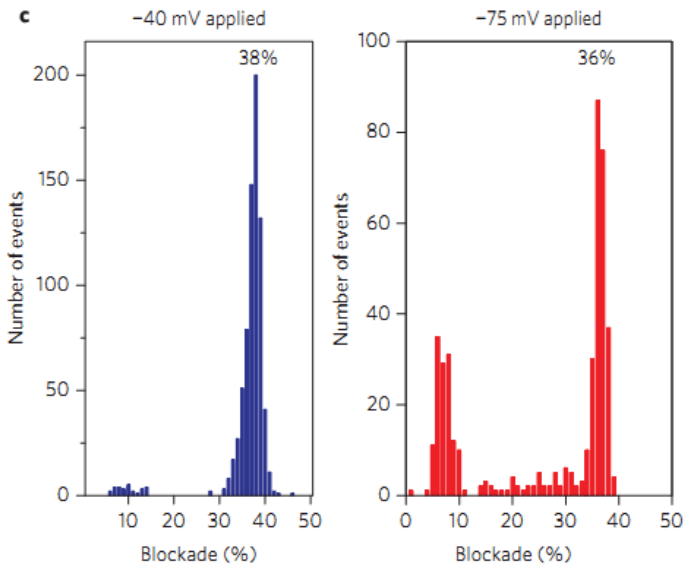

Fig. 8 (c) The percentage of current blockade caused by linear plasmid double stranded DNA under $-40 \mathrm{mV}$ and $-75 \mathrm{mV}$.

The process is showedin Fig. 5 and Fig. 6.

\section{3. membrane-adapted phi29 motor protein nanopore}

The previous mentioned protein nanopores have channels that only allow the movement of small molecules and single stranded DNA or RNA. In this section, we will introduce the membrane-adapted phi29 motor protein nanopore, which allows double stranded DNA to enter the virus during maturation and to exit during infection. It contains a connector protein with a channel whose width is between $3.6 \mathrm{~nm}$ and $6 \mathrm{~nm}$. David Wendell, Peng Jing et al. [7] have modified this connector protein. And when it is reconstituted into liposomes and inserted into planar lipid bilayers, it allows the translocation of double stranded DNA. It uses 6 packaging RNA strands to gear the machine.

The motor of bacteriophage phi29 uses 1 ATP to package 2 or 2.5 base pairs of DNA. The structure of the protein is shown in Fig. 7. It has a truncated cone structure, and termed a connector that allows double stranded DNA to enter during maturation and to exit during infection. The connector has a central channel consisting of 12 GP10 protein subunits. The phi29 connector is ideally suited for undergoing nanopore sequencing because its available crystal structure allows for explicit engineering. Moreover, procedures for large scale production and purification of the phi29 connector have already been developed [7].
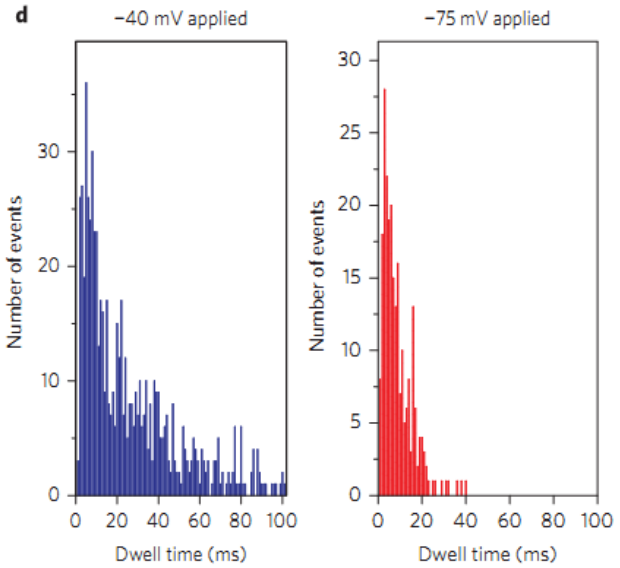

Fig. 8 (d) The dwell time caused by linear plasmid double stranded DNA under $-40 \mathrm{mV}$ and $-75 \mathrm{mV}$ [7].

After modifying the phi29 connector, reconstituting the connector into liposomes and incorporating the connector into planar lipid membranes, the desired product has been prepared. (detailed procedure in reference [7]) when linear DNA plasmid passes through the nanopore, its translocation induced numerous current blockades which led to the current jump of single connector insertion to be transiently reduced by $25-45 \%$. At the same time, blockade events in the range of 5-15\% are occasionally detected, which is due to the interactions of connector pores with lipids or lipid micelles rather than DNA translocation because they were also found in the absence of DNA. This kind of blockades are minimized when diluted connector reconstituted liposomes are used or a lower transmembrane voltage is applied. And when DNA was premixed with buffer before connector insertion, the DNA blockades were detected immediately after the first insertion took place. Details are shown in Fig. 8.

The blockade rates were affected by two factors: DNA concentration and transmembrane voltage. And the dwell time of DNA translocation was affected by applied the size of DNA and voltage. Moreover, the DNA translocation rate was affected by the number of inserted connectors [7].

This engineered form of the GP10 connector is incorporated into a lipid bilayer, forming a highly conductive nanopore. The translocation of the linear 
plasmid DNA may be confirmed by evaluating the changes of the conductance and performing Q-PCR analysis. This work provides other researchers with a tool or a system for future electrophysiology studies of the phi29 DNA packaging motor. Moreover, this kind of connector is easy to engineer and extremely reproducible because it is a biological nanopore, which makes it suitable for future nanotechonological and biomedical applications.

\section{References}

1.Deamer DW, and Akeson M. Nanopores and nucleic acids: prospects for ultrarapid sequencing, Trends in biotechnology. 2000; 18:147-151.http://dx.doi.org/10.1016/S01677799(00)01426-8

2. Marziali A, and Akeson M. New DNA sequencing methods. Annual review of biomedical engineering. 2001; 3:195-223.http:// dx.doi.org/10.1146/annurev.bioeng.3.1.195

3. Song L, Hobaugh M R, Shustak C, Cheley S, Bayley H, and Gouaux JE. Structure of staphylococcal $\alpha$-hemolysin, a heptameric transmembrane pore. Science 1996; 274:1859.http:// dx.doi.org/10.1126/science.274.5294.1859
4. Akeson M, Branton D, Kasianowicz JJ, Brandin E, and Deamer DW. Microsecond time-scale discrimination among polycytidylic acid, polyadenylic acid, and polyuridylic acid as homopolymers or as segments within single RNA molecules. Biophysical journal.1999; 77:3227-3233.http://dx.doi.org/10.1016/S0006-3495(99)77153-5

5. Derrington IM, Butler TZ, Collins MD, Manrao E, Pavlenok M, Niederweis M, and Gundlach JH. Nanopore DNA sequencing with MspA, Proceedings of the National Academy of Sciences 2010; 107:16060-16065.http://dx.doi.org/10.1073/pnas.1001831107

6. Butler TZ, Pavlenok M, Derrington IM, Niederweis M, Gundlach JH. Single-molecule DNA detection with an engineered MspA protein nanopore, Proceedings of the National Academy of Sciences. 2008; 105:20647.http://dx.doi.org/10.1073/pnas.0807514106

7. Wendell D, Jing P, Geng J, Subramaniam V, Lee TJ, Montemagno C, and Guo P. Translocation of double-stranded DNA through membrane-adapted phi29 motor protein nanopores. Nature nanotechnology 2009; 4:765-772.http://dx.doi.org/10.1038/ nnano.2009.259

Copyright:(c) $2012 \mathrm{~J}$. Shen, et al. This is an openaccess article distributed under the terms of the Creative Commons Attribution License, which permits unrestricted use, distribution, and reproduction in any medium, provided the original author and source are credited. 